\title{
Resveratrol regulates type II collagen and COX-2 expression via the ERK, p38 and Akt signaling pathways in rabbit articular chondrocytes
}

\author{
SEONG-HUI EO ${ }^{1}$, HONG-SIK CHO ${ }^{2-4}$ and SONG-JA KIM ${ }^{1}$ \\ ${ }^{1}$ Department of Biological Sciences, College of Natural Sciences, Kongju National University, Gongju, Chungnam 314-701, \\ Republic of Korea; ${ }^{2}$ The University of Tennessee Health Science Center, Memphis, TN 38163; \\ ${ }^{3}$ Department of Orthopaedic Surgery, Campbell Clinic, Memphis, TN 38163; \\ ${ }^{4}$ Veterans Affairs Medical Center, Memphis, TN 38163, USA
}

Received August 22, 2013; Accepted December 27, 2013

DOI: $10.3892 /$ etm.2014.1484

\begin{abstract}
Resveratrol, a naturally occurring polyphenolic phytoalexin antioxidant compound present in grapes and red wine, has been reported to induce various biochemical responses. It has been shown to possess anti-aging, anti-inflammatory and anti-proliferative activities in several cell types. However, the effects of resveratrol in normal cells, including chondrocytes, have not yet been clearly elucidated. The aim of the present study was to evaluate the effects of resveratrol on differentiation and inflammation in rabbit articular chondrocytes and to investigate the underlying mechanism of action. Rabbit articular chondrocytes were treated with $20 \mu \mathrm{M}$ resveratrol for different time periods or with various concentrations of resveratrol for $24 \mathrm{~h}$. It was observed that the expression levels of type II collagen and sulfated proteoglycan, as determined by western blot analysis and Alcian blue staining, respectively, increased following treatment with resveratrol in a concentration-dependent manner at concentrations up to $20 \mu \mathrm{M}$ and then decreased at higher concentrations. The expression levels of cyclooxygenase (COX-2) and prostaglandin $\mathrm{E}_{2}\left(\mathrm{PGE}_{2}\right)$ began to increase at 10 min after the addition of resveratrol, reached peak levels at $3 \mathrm{~h}$ and decreased from the peak level thereafter, as determined by western blot analysis and $\mathrm{PGE}_{2}$ assay, respectively. It was also demonstrated that resveratrol caused phosphorylation of mitogen-activated protein kinase proteins [extracellular signal-regulated kinases (ERK), p38 and c-Jun N-terminal
\end{abstract}

Correspondence to: Professor Song-Ja Kim, Department of Biological Sciences, College of National Sciences, Kongju University, 182 Sinkwan-dong, Gongju, Chungnam 314-701, Republic of Korea

E-mail:ksj85@kongju.ac.kr

Key words: chondrocytes, resveratrol, type II collagen, cyclooxygenase-2 kinases (JNK)] and Akt in rabbit articular chondrocytes. The inhibition of ERK, p38 kinase, phosphoinositide 3-kinase (PI3K) and Akt with PD98059, SB203580, LY294002 and triciribine, respectively, suppressed resveratrol-induced type II collagen and COX-2 expression. However, inhibition of JNK with SP600125 produced no clear changes in the expression levels of type II collagen and COX-2. The results suggest that resveratrol in articular chondrocytes stimulates differentiation and inflammation via the ERK, p38 and Akt signaling pathways.

\section{Introduction}

Chondrocytes in articular cartilage are differentiated from mesenchymal cells during embryonic development (1-3). The differentiated chondrocytes are able to proliferate and undergo hypertrophic maturation. Cartilage is composed of a dense extracellular matrix, made up from macromolecules such as type II collagen, sulfated proteoglycan and fibronectin (4). This biosynthetic composition of chondrocytes is maintained during complex biological processes, including cartilage development, differentiation and repair. However, the differentiated chondrocyte phenotype is unstable in culture and destroyed in degenerative diseases, such as osteoarthritis (OA) and rheumatoid arthritis (RA) (5-9).

Cyclooxygenase $(\mathrm{COX})$ is an enzyme that catalyzes the conversion of arachidonic acid to prostaglandin $\mathrm{H}_{2}$, the precursor of a variety of biologically active mediators such as prostaglandin $\mathrm{E}_{2}\left(\mathrm{PGE}_{2}\right)$, prostacyclin and thromboxane A2 $(10,11)$. Two isoforms of COX are COX-1 and COX-2. COX-1 is constitutively expressed in a wide variety of tissues, is ubiquitous in its distribution, and is thought to be involved in tissue homeostasis and maintenance of the levels of prostaglandins. COX-2 is an enzyme induced by pro-inflammatory cytokines, tumor promoters, oncogenes and growth factors, and is involved mainly in the regulation of inflammatory responses in numerous types of cell, such as monocytes, fibroblasts and endothelial cells $(2,12)$.

Resveratrol $\left(\mathrm{C}_{14} \mathrm{H}_{12} \mathrm{O}_{3} ; 3,5,4\right.$ '-trihydroxy-trans-stilbene) was first identified in the roots of white hellebore 
(Veratrum grandiflorum) in 1940 (13). Resveratrol is a natural polyphenolic compound that is also found in the skin of red grapes, cranberries and peanuts, and the root extracts of the weed Polygonum cuspidatum (14-16). Numerous signaling pathways involving resveratrol have been evaluated and a number of its targets and mechanisms of action have been identified. It has been reported that resveratrol has antitumor activity and immunomodulatory, antioxidative and anti-inflammatory functions, as well as numerous biological activities. Resveratrol has been shown to exhibit in vitro as well as in vivo chemopreventive and chemotherapeutic activities (15,17-20).

Elmali et al observed a significant protective effect of resveratrol injections on articular cartilage degradation in rabbit models for OA and RA via histological analysis in vivo (21). Resveratrol has been demonstrated to suppress aging by activating the SIRT1 gene, which suppresses cell apoptosis (22-26). In human articular chondrocytes, Czaki et al elucidated anti-apoptotic and anti-inflammatory regulatory mechanisms mediated by resveratrol (27). In human articular chondrocytes, resveratrol together with curcumin was shown to suppress the apoptosis induced by IL-1 $\beta$ through stimulation of the mitogen-activated protein kinase (MAPK) signaling pathway (27).

However, the effects of resveratrol on differentiation and the inflammatory response in normal cells, including chondrocytes, and the mechanism by which resveratrol acts are not clearly understood. As a result, the present study was conducted to investigate the effects of resveratrol on differentiation and the inflammatory response of rabbit chondrocytes and to analyze the subsequently regulated intracellular signal transduction pathways.

\section{Materials and methods}

Reagents and antibodies. Resveratrol was purchased from Sigma-Aldrich (St. Louis, MO, USA). The resveratrol was diluted in sterile dimethylsulfoxide (Sigma-Aldrich; final concentration in the medium was $>1 \%$ ) and stored at $-20^{\circ} \mathrm{C}$. Dulbecco's modified Eagle's medium (DMEM) and fetal bovine serum (FBS) were purchased from Invitrogen (Burlington, ON, Canada). Streptomycin, penicillin and SP600125 were obtained from Sigma-Aldrich. SB203580 (SB), PD98059 (PD), LY294002 (LY) and triciribine (TB) were purchased from Calbiochem (San Diego, CA, USA). Type II collagen, actin, COX-2 and pERK antibodies were obtained from Santa Cruz Biotechnology Inc. (Santa Cruz, CA, USA) and pAkt, p38 and pJNK were from Cell Signaling Technology (Danvers, MA, USA).

Cell culture. Rabbit articular chondrocytes were isolated from the cartilage of two-week-old New Zealand white rabbits (KOATECH, Pyeongtaek-si, Gyeonggi-do, Korea) using enzymatic digestion, as described previously (28). The cartilage slices were dissociated enzymatically for $6 \mathrm{~h}$ in $0.2 \%$ collagenase type II (381 U/mg solid; Sigma-Aldrich) in DMEM. Following collection of individual cells by brief centrifugation at $230 \mathrm{x} \mathrm{g}$ for $10 \mathrm{~min}$ and $20^{\circ} \mathrm{C}$, the cells were suspended in DMEM supplemented with $10 \%$ (v/v) FBS, $50 \mu \mathrm{g} / \mathrm{ml}$ streptomycin and $50 \mathrm{U} / \mathrm{ml}$ penicillin. The cells were then plated on culture dishes at a density of $5 \times 10^{4}$ cells $/ \mathrm{cm}^{2}$. The medium was changed every two days, and the cells reached confluence after approximately five days. After three days the cell cultures were treated with resveratrol. The following pharmacological agents were added $1 \mathrm{~h}$ prior to the addition of resveratrol: SB to inhibit p38 kinase, PD to inhibit ERK, and LY and TB to inhibit phosphoinositide 3-kinase (PI3K) and Akt, respectively. The study was approved by the Ethics Committee of Kongju National University (Gongju, Republic of Korea; IRB no. 2011-2).

Western blot analysis. Whole cell lysates were prepared by extracting proteins using a cold radioimmunoprecipitation assay buffer [50 mM Tris- $\mathrm{HCl}, \mathrm{pH} 7.4 ; 150 \mathrm{mM} \mathrm{NaCl}$; $1 \%$ Nonidet P-40; and $0.1 \%$ sodium dodecylsulfate (SDS); supplemented with protease inhibitors $(10 \mu \mathrm{g} / \mathrm{ml}$ leupeptin, $10 \mu \mathrm{g} / \mathrm{ml}$ pepstatin $\mathrm{A}, 10 \mu \mathrm{g} / \mathrm{ml}$ aprotinin and $1 \mathrm{mM}$ 4-(2-aminoethyl)benzenesulfonyl fluoride and phosphatase inhibitors $\left(1 \mathrm{mM} \mathrm{NaF}\right.$ and $\left.1 \mathrm{mM} \mathrm{Na} \mathrm{VO}_{4}\right)$ ] obtained from Sigma-Aldrich. The lysates were size-fractionated by SDS-polyacrylamide gel electrophoresis and transferred to a nitrocellulose (NC) membrane (Whatman Schleicher and Schuell, Dassel, Germany). The NC sheet was then blocked with 5\% non-fat dry milk in Tris-buffered saline. Antibodies against type II collagen, COX-2, pp38, pERK, pJNK and Akt were used for probing corresponding NC blots overnight at $4^{\circ} \mathrm{C}$. The membranes were then washed three times with Tris-buffered saline/Tween-20 and incubated with horseradish peroxidase-conjugated secondary antibody (Sigma-Aldrich) for $2 \mathrm{~h}$ followed by exposure in an LAS-4000 imager (Fujifilm Corp., Tokyo, Japan) according to the manufacturer's instructions. The experimental results were transformed into in numerical values using Image J 1.41 (Software Inquiry, Quebec, Canada).

$P G E_{2}$ assay. $\mathrm{PGE}_{2}$ production in the articular chondrocytes was determined by measuring the levels of cellular and secreted $\mathrm{PGE}_{2}$ with an assay kit purchased from Assay Design Inc. (Ann Arbor, MI, USA). A PGE 2 linked immunosorbent assay kit was purchased from Amersham Pharmacia Biotech (Piscataway, NJ, USA). Briefly, the chondrocytes were seeded in standard 96-well microtiter plates at a density of $2 \times 10^{4}$ cells/well and treated with various reagents, such as resveratrol, for $1 \mathrm{~h}$ prior to treatment with SB, PD, LY and TB for $3 \mathrm{~h}$ after treatment with resveratrol in the absence or presence of inhibitors (SB, PD, LY and TB). The amount of $\mathrm{PGE}_{2}$ present in total cell lysates was quantified according to the kit manufacturer's instructions. Levels were calculated against a standard curve of $\mathrm{PGE}_{2}$.

Immunofluorescence staining. The expression levels and distribution of type II collagen and COX-2 in the rabbit articular chondrocytes were determined by indirect immunofluorescence microscopy, as described previously (28). Rabbit chondrocytes were fixed with $3.5 \%$ paraformaldehyde in phosphate-buffered saline (PBS) for $15 \mathrm{~min}$ at room temperature, and permeabilized and blocked with $0.1 \%$ Triton $\mathrm{X}-100$ and $5 \%$ fetal calf serum in PBS for $30 \mathrm{~min}$. The fixed cells were washed three times with PBS and incubated for $2 \mathrm{~h}$ with antibodies against type II collagen (Santa Cruz Biotechnology, 
A

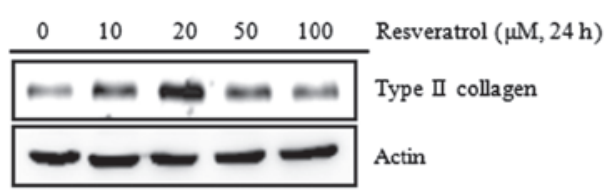

C

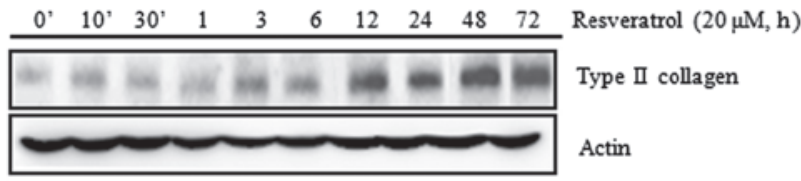

B

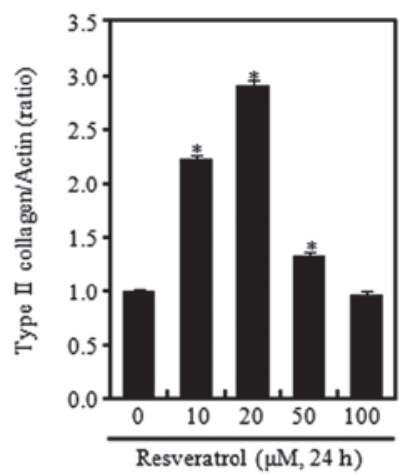

D

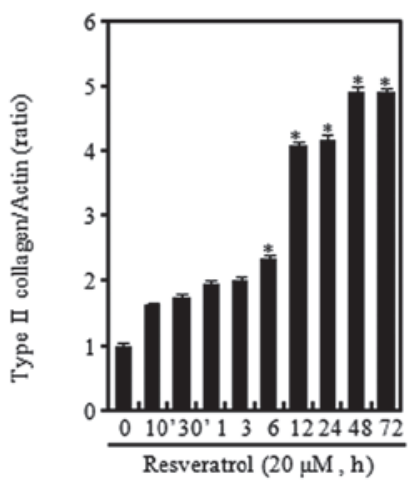

Figure 1. Resveratrol regulates type II collagen expression in rabbit articular chondrocytes. Rabbit articular chondrocytes were treated with various concentrations of resveratrol for the indicated time periods. (A and C) The expression levels of type II collagen were analyzed by western blot analysis. Actin was used as a loading control. (B and D) The relative amounts of type II collagen were quantified by densitometric measurement (ImageJ). The data represent a typical experiment, whereby similar results were obtained from three experiments. ${ }^{*} \mathrm{P}<0.05$, compared with untreated cells.

Inc.) or COX-2 (Cayman Chemical, Ann Arbor, MI, USA). The cells were washed and incubated with rhodamine or fluorescein-conjugated secondary antibodies for $1 \mathrm{~h}$, washed with PBS, and observed under a fluorescence microscope (Olympus, Tokyo, Japan).

Immunohistochemical staining. The cartilage explants $\left(125 \mathrm{~mm}^{3}\right)$ were fixed in $4 \%$ paraformaldehyde in PBS for $24 \mathrm{~h}$ at $4^{\circ} \mathrm{C}$, washed with PBS, dehydrated with graded ethanol, embedded in paraffin and sectioned into $4-\mu \mathrm{m}$ slices as described previously (28). The sections were stained by the standard procedures using antibodies against type II collagen or COX-2, and visualized by development with an EnVisionTM+ kit purchased from Dako (Carpinteria, CA, USA) following the manufacturer's instructions.

Determination of chondrocyte phenotype. The cells were fixed with $95 \%$ methanol at $-20^{\circ} \mathrm{C}$ for 2 min and stained with $0.1 \%$ Alcian blue (Sigma Aldrich) in $0.1 \mathrm{M} \mathrm{HCl}$ overnight. The chondrocytes were washed three time with PBS buffer and $6 \mathrm{M}$ guanidine $\mathrm{HCl}$ was added for $6 \mathrm{~h}$. Production of sulfated proteoglycan was measured at $620 \mathrm{~nm}$ by an enzyme-linked immunosorbent assay.

Data analysis and statistics. The results are expressed as the mean \pm standard deviation. The values were calculated from the specified number of determinations. The significance of the differences between the experimental and control groups was assessed by one-way ANOVA. A value of $\mathrm{P}<0.05$ was considered to indicate a statistically significant difference.

\section{Results}

Resveratrol regulates the differentiation of chondrocytes. An aim of this study was to determine whether resveratrol regulates the expression of type II collagen and sulfated proteoglycan in rabbit articular chondrocytes (Fig. 1). Various concentrations of resveratrol were tested, and at low concentrations $(\leq 20 \mu \mathrm{M})$ it was found that the expression levels of type II collagen gradually increased in a concentration-dependent manner when compared with those in the control chondrocytes without resveratrol treatment. However, at a high concentration $(100 \mu \mathrm{M})$, the expression level of type II collagen was observed to be reduced (Fig. 1A). When cells were treated with $20 \mu \mathrm{M}$ resveratrol, type II collagen expression levels were increased in a time-dependent manner (Fig. 1C). The expression levels were determined using ImageJ software (Fig. 1B and D). Alcian blue staining was used to identify the levels of sulfated proteoglycan, which is an extracellular substrate molecule commonly used as another marker protein for differentiation of chondrocytes. Similar to type II collagen expression, the Alcian blue staining results showed that the levels of proteoglycan increased compared with those in the control when the chondrocytes were treated with $20 \mu \mathrm{M}$ resveratrol, while they were less elevated when the chondrocytes were treated with $100 \mu \mathrm{M}$ resveratrol (Fig. 2A). Moreover, it was observed that the changes in the proteoglycan levels in relation to time also started to increase from $24 \mathrm{~h}$ after the addition of resveratrol, similar to the effect on the expression of type II collagen (Fig. 2B). In order to verify the aforementioned results at chondrocytic cellular and tissue levels, immunofluorescence 
A

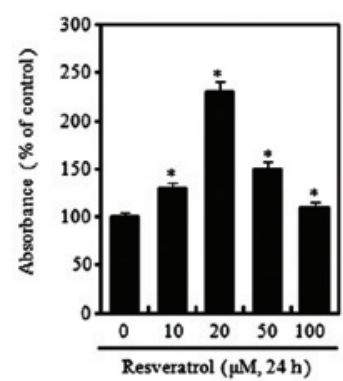

C

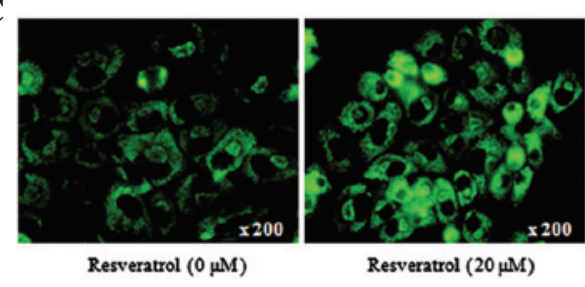

B

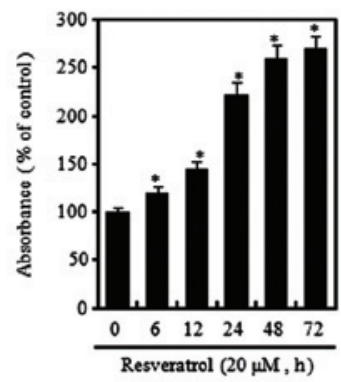

D

Resveratrol $(0 \mu \mathrm{M})$

Resveratrol $(20 \mu \mathrm{M})$

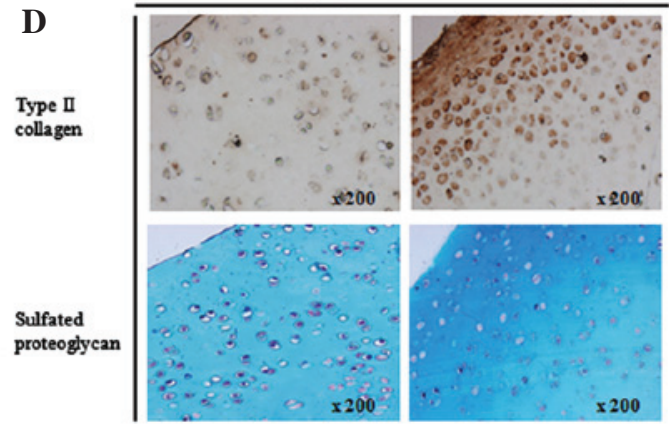

Figure 2. Resveratrol (Res) regulates differentiation in rabbit articular chondrocytes. Rabbit articular chondrocytes were untreated or treated (A) with various concentrations of resveratrol for $24 \mathrm{~h}$ or (B) with $20 \mu \mathrm{M}$ resveratrol for the specified time periods. (C) Expression of type II collagen was determined by immunofluorescence staining and (D, upper panel) immunohistochemical staining (magnification, x200). (D, lower panel) Sulfated proteoglycan was detected in tissue by Alcian blue staining. The data represent a typical experiment, whereby similar results were obtained from four experiments. ${ }^{*}<0.05$, compared with untreated cells.

and immunohistochemical staining assays were performed to identify the levels of type II collagen and proteoglycan. As a result of performing immunofluorescence staining using type II collagen antibody following treatment with $20 \mu \mathrm{M}$ resveratrol for $24 \mathrm{~h}$, increased expression levels of type II collagen in resveratrol-treated cells were confirmed (Fig. 2C). Moreover, at the cartilaginous tissue level, increased expression levels of type II collagen were confirmed (Fig. 2D, upper panel). In addition, the results of performing Alcian blue staining following treatment of the cartilaginous tissue with $20 \mu \mathrm{M}$ resveratrol for $24 \mathrm{~h}$ showed increased levels of proteoglycan in the resveratrol-treated tissues (Fig. 2D, lower panel). In general reference to the aforementioned results, it was found that chondrocytic differentiation was regulated differentially according to the concentration of resveratrol, and inducement of chondrocytic differentiation was identified after $24 \mathrm{~h}$ of treatment with resveratrol at a low concentration $(20 \mu \mathrm{M})$.

Resveratrol induces an inflammatory response in chondrocytes. Previous studies have shown that the expression of COX-2 increases the levels of $\mathrm{PGE}_{2}$ and that $\mathrm{PGE}_{2}$ induces various inflammatory reactions $(10,29)$. In the present study, chondrocytes were treated with resveratrol at $20 \mu \mathrm{M}$ for different time periods (Fig. 3A and B) or with various concentrations of resveratrol for $3 \mathrm{~h}$ (Fig. 3C and D). Stimulation of cells with resveratrol induced a marked increase in COX-2 expression levels, which was apparent within $3 \mathrm{~h}$ after treatment with resveratrol. The COX-2 expression levels peaked at $3 \mathrm{~h}$ and the subsequent reduction was detectable for up to $72 \mathrm{~h}$ (Fig. 3A and B). Concentration-dependent increases in COX-2 expression levels were measured by western blot analysis and densitometric analysis (Fig. 3C and D). In order to find out more clearly whether or not resveratrol induces an inflammatory response in chondrocytes, a $\mathrm{PGE}_{2}$ assay was performed to evaluate the levels of $\mathrm{PGE}_{2}$, which is a product of $\mathrm{COX}-2$, and changes in the expression levels of COX-2 at chondrocytic cellular and tissue levels were identified through immunofluorescence and immunohistochemical staining (Fig. 4). As a result of performing the $\mathrm{PGE}_{2}$ assay following the treatment of chondrocytes with resveratrol at different concentrations and time periods, it was possible to verify that the levels of $\mathrm{PGE}_{2}$ greatly increased at $3 \mathrm{~h}$ after treatment with $20 \mu \mathrm{M}$ resveratrol but had begun to decrease by $6 \mathrm{~h}$, in a similar manner to COX-2 (Fig. 4A). Moreover, it was confirmed that the $\mathrm{PGE}_{2}$ production levels were increased by resveratrol in a concentration-dependent manner (Fig. 4B). In order to identify the levels of COX-2 expression at the chondrocytic cell and tissue levels, immunofluorescence and immunohistochemical staining was conducted. Increased COX-2 expression levels were observed in the cells and tissues treated with resveratrol (Fig. 4C and D). The aforementioned results signify that resveratrol induces an inflammatory response in chondrocytes.

Resveratrol increases the activation of MAPK and Akt. To investigate through which signal transduction system the chondrocytic differentiation and inflammatory response due to resveratrol are regulated, the activation of MAPK and PI3K was examined. The MAPK and PI3K signal transduction pathways are closely associated with the regulation of chondrocytic differentiation and the inflammatory response (Fig. 5). The results confirmed an increase in activation of MAPK-related proteins (ERK, p38 and JNK) and Akt due to resveratrol (Fig. 5A). To study the changes in the expression levels of the proteins associated with cellular signal transduction according 
A

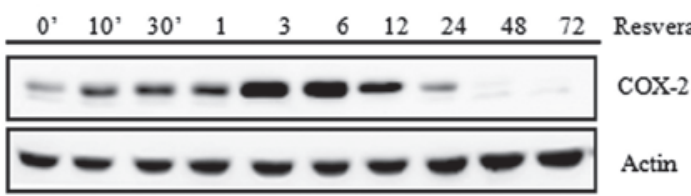

C

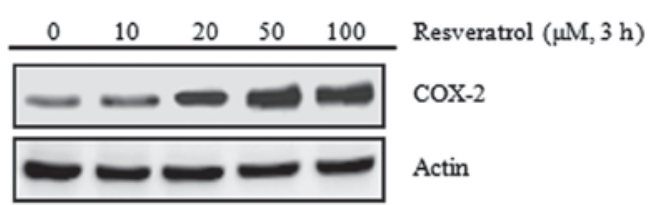

B

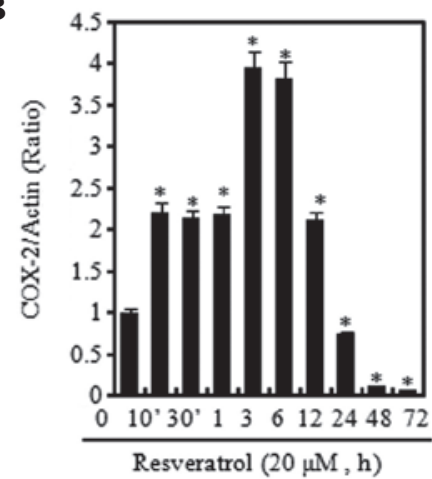

D

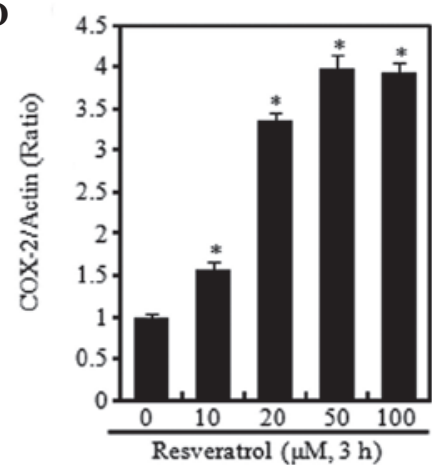

Figure 3. Resveratrol regulates the expression of COX-2 in rabbit articular chondrocytes. Rabbit articular chondrocytes were treated (A and B) with $20 \mu \mathrm{M}$ resveratrol for 0-72 $\mathrm{h}$ or (C and D) with 10-100 $\mu \mathrm{M}$ Res for $3 \mathrm{~h}$. (A and C) Expression levels of COX-2 were determined by western blot analysis. Actin was used as a loading control. (B and D) The relative amounts of COX-2 were quantified by densitometric measurement (with ImageJ software). The data represent a typical experiment, whereby similar results were obtained from three experiments. ${ }^{*} \mathrm{P}<0.05$, compared with untreated cells. COX-2, cyclooxygenase- 2 .

A

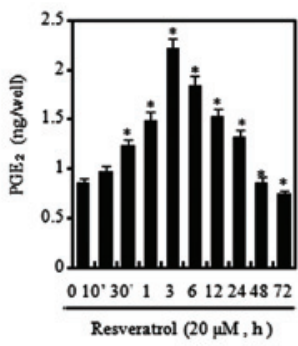

C

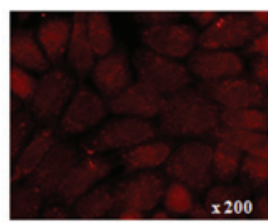

Resveratrol $(0 \mu \mathrm{M})$

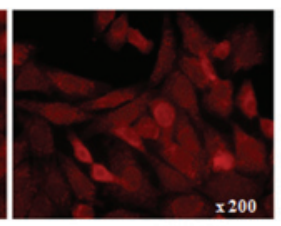

Resveratrol $(20 \mu \mathrm{M})$
B

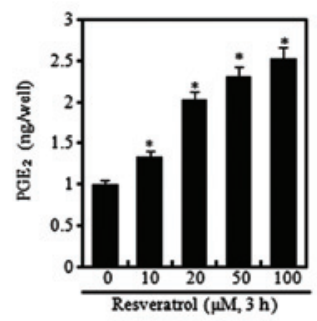

D

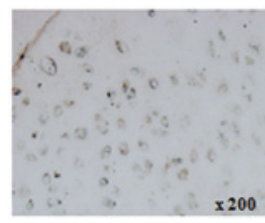

Resveratrol $(0 \mu \mathrm{M})$

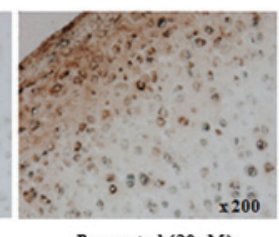

Resveratrol $(20 \mu \mathrm{M})$

Figure 4. Resveratrol (Res) regulates the inflammatory response in rabbit articular chondrocytes. (A) Rabbit articular chondrocytes were untreated or treated with $20 \mu \mathrm{M}$ resveratrol for the indicated time periods. (B) Rabbit articular chondrocytes were untreated or treated with the indicated concentrations of resveratrol for $3 \mathrm{~h}$. $\mathrm{PGE}_{2}$ production was measured using a $\mathrm{PGE}_{2}$ assay kit. (C) Rabbit articular chondrocytes or (D) cartilage explants were untreated or treated with $20 \mu \mathrm{M}$ resveratrol for $24 \mathrm{~h}$. Expression of COX-2 was detected by immunofluorescence staining. The data represent a typical experiment, whereby similar results were obtained from three experiments. ${ }^{*} \mathrm{P}<0.05$, compared with untreated cells. $\mathrm{PGE}_{2}$, prostaglandin $\mathrm{E}_{2}$.

to treatment time, western blot analysis was performed following treatment with $20 \mu \mathrm{M}$ of resveratrol for various time periods up to $72 \mathrm{~h}$. The results showed that the activation levels of MAPK-related proteins (ERK, p38 and JNK) started to increase from $6 \mathrm{~h}$ and those of Akt started to increase from $24 \mathrm{~h}$ after the resveratrol treatment (Fig. 5B). Such results signify that chondrocytic differentiation and the inflammatory response induced by resveratrol are associated with activation of MAPK and Akt. Accordingly, inhibitors of MAPK-related proteins (ERK inhibitor, PD; p38 inhibitor, SB; JNK inhibitor, $\mathrm{SP}$ ), TB (an inhibitor of Akt), and LY (an inhibitor of PI3K; the $\mathrm{PI} 3 \mathrm{~K}$ pathway is an upstream signal transduction pathway for Akt), were used to clearly identify the signal transduction pathways that are regulated by resveratrol. The chondrocytes first underwent pretreatment $1 \mathrm{~h}$ prior to resveratrol treatment to block the ERK, p38, JNK and Akt signaling pathways and 
A

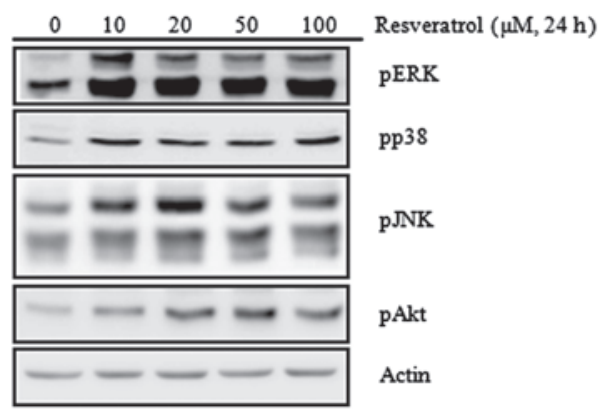

$\mathbf{B}$

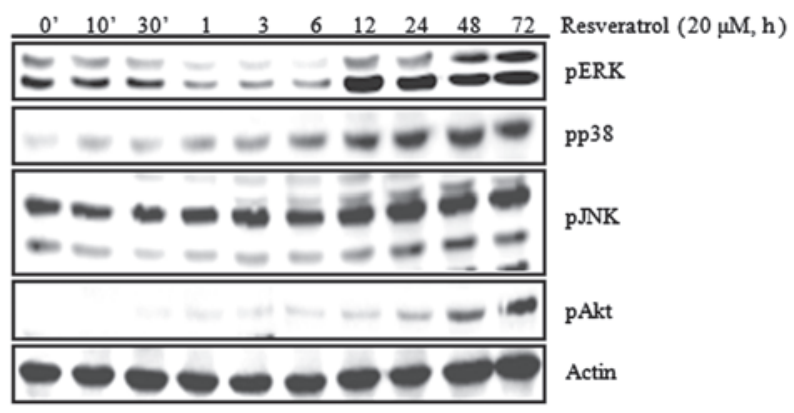

Figure 5. Resveratrol induces the activities of MAPKs and pAkt in rabbit articular chondrocytes. Rabbit articular chondrocytes were untreated or treated (A) with the indicated concentrations of resveratrol for $24 \mathrm{~h}$, or (B) with $20 \mu \mathrm{M}$ resveratrol for the indicated time periods. Expression of the MAPK proteins (pERK, pp38 and pJNK) and pAkt was determined by western blot analysis. Expression of actin was used as a loading control. The data represent the results of a typical experiment from at least four independent experiments. MAPK, mitogen-activated protein kinase; ERK, extracellular signal-regulated kinase; JNK, phosphorylated c-Jun N-terminal kinase.

A

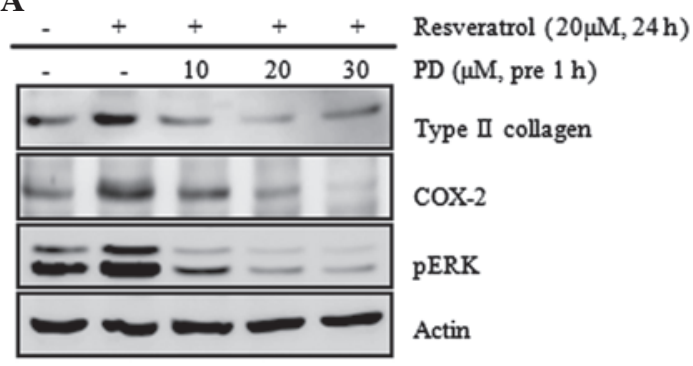

C

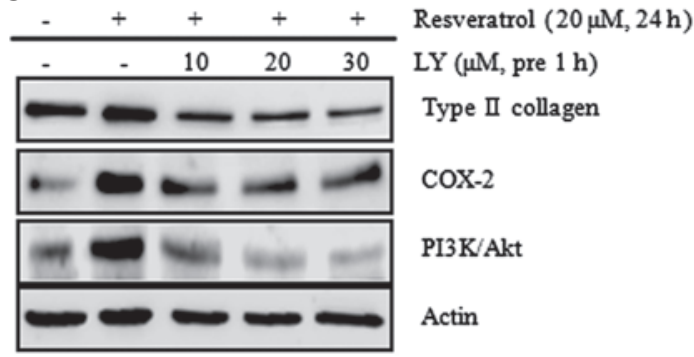

B

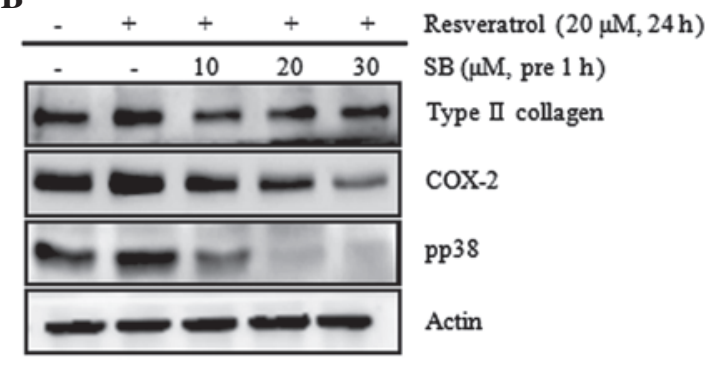

D

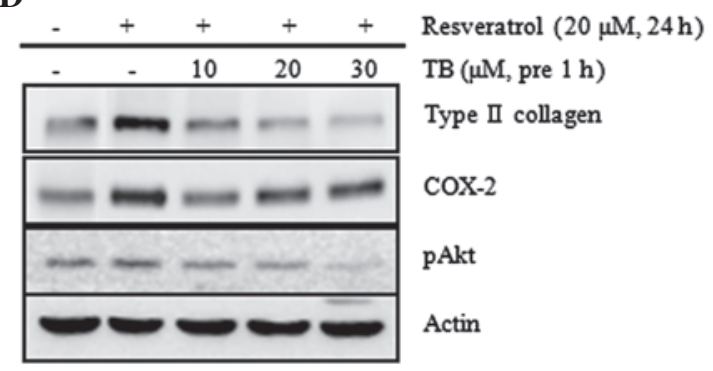

Figure 6. Resveratrol induces type II collagen and COX-2 expression via the ERK, p38 and Akt signaling pathways in rabbit articular chondrocytes. Rabbit articular chondrocytes were untreated or treated with the indicated concentrations of the inhibitors: (A) PD, an inhibitor of ERK; (B) SB, an inhibitor of p38; (C) LY, an inhibitor of PI3K/Akt; or (D) TB, an inhibitor of Akt for $1 \mathrm{~h}$ and then treated with $20 \mu \mathrm{M}$ resveratrol for $24 \mathrm{~h}$. Expression of pERK, pp38, pAkt, type II collagen and COX-2 was detected by western blot analysis. Expression of actin was used as the loading control. The data represent the results of a typical experiment from at least four independent experiments. PD, PD98059; SB, SB203580; LY, LY294002; TB, triciribine; COX-2, cyclooxygenase-2; ERK, extracellular signal-regulated kinase; PI3K, phosphoinositide 3-kinase.

were then treated with resveratrol, following which changes to chondrocytic differentiation and the inflammatory response proteins were studied through western blot analysis, immunofluorescence staining, Alcian blue staining and $\mathrm{PGE}_{2}$ assay (Fig. 6 and 7). The results of these assays showed that the resveratrol-induced increases in the expression levels of type II collagen and COX-2 were attenuated by treatment with $\mathrm{SB}$, PD, LY and TB (Fig. 6). However, no changes to the levels of type II collagen and COX-2 expression due to SP treatment were observed (data not shown). Following verification using Alcian blue staining, immunofluorescence staining and $\mathrm{PGE}_{2}$ assay methods, the resveratrol-induced increases in the levels of proteoglycan and expression levels of type II collagen and COX-2 were observed to be similarly attenuated by PD, SB,
TB and LY treatment (Fig. 7). Such results indicate that the differentiation and inflammatory response induced by resveratrol are mediated through the ERK, p38 and Akt signal transduction pathways.

\section{Discussion}

Resveratrol, one of the major stilbenes, has a structure that is related to the synthetic estrogen diethylstilbestrol. It comprises two phenol rings linked by a styrene double bond and exists in two isoforms $(19,30)$. Resveratrol has been demonstrated to process anticancer, anti-aging, anti-inflammatory and neuroprotective activities (31). Resveratrol has also been found to exhibit diverse biological effects; it induces MMP-9 expression 
A
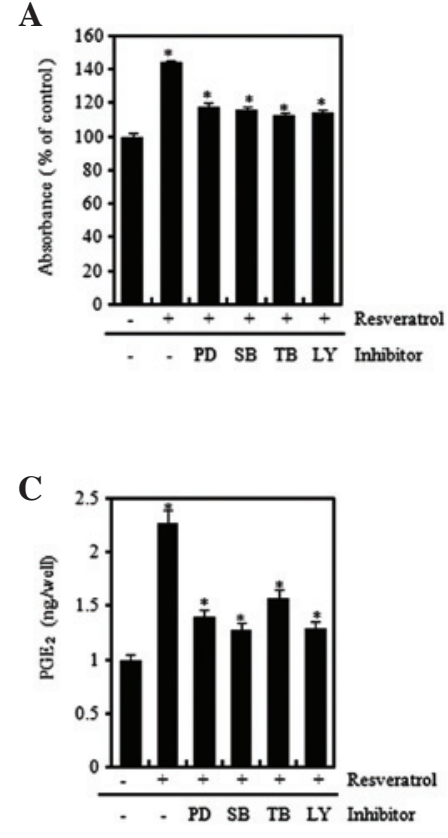

B
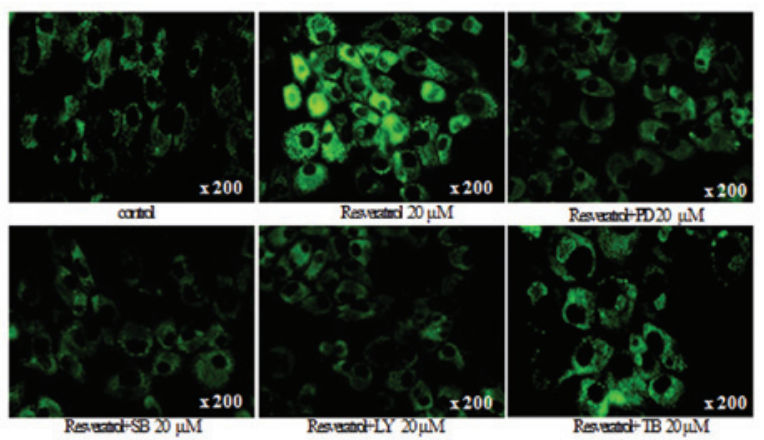

D
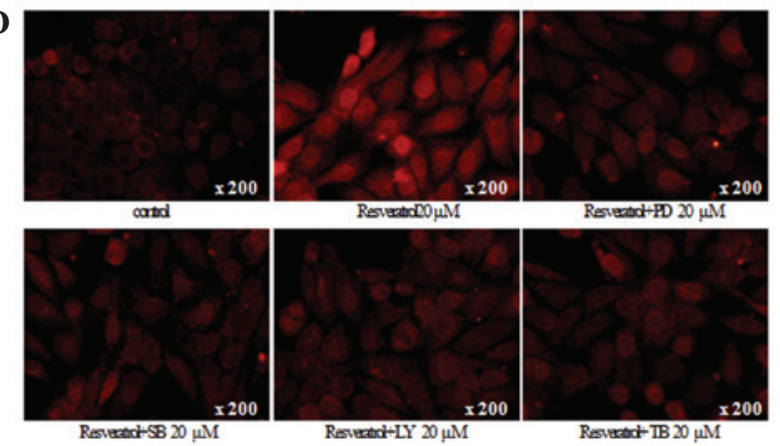

Figure 7. Resveratrol (Res) induces differentiation and inflammation in rabbit articular chondrocytes. (A) Rabbit articular chondrocytes were untreated or treated with the $20 \mu \mathrm{M}$ of inhibitors (PD98059, SB203580, LY294002) and triciribine (TB) for $1 \mathrm{~h}$ and then treated with $20 \mu \mathrm{M}$ Res for $24 \mathrm{~h}$. Accumulation of sulfated proteoglycan was determined by Alcian blue staining. Expression of (B) type II collagen and (D) COX-2 was determined by immunofluorescence staining (magnification, x200). (C) Rabbit articular chondrocytes were untreated or treated with the $20 \mu$ M of inhibitors (PD98059, SB203580, LY294002) and triciribine (TB) for $1 \mathrm{~h}$ and then treated with $20 \mu \mathrm{M}$ Res for $3 \mathrm{~h}$. ${ }^{*} \mathrm{P}<0.05$, compared with untreated cells. PD, PD98059; SB, SB203580; LY, LY294002; TB, triciribine; COX-2, cyclooxygenase-2; $\mathrm{PGE}_{2}$, prostaglandin $\mathrm{E}_{2}$.

and cell migration via the p38 kinase and PI3K pathway in HT1080 human fibrosarcoma cells (32) and induces differentiation via reduction of the expression of MMPs; this regulation is mediated by the p38 and JNK pathway in HTB94 human chondrosarcoma cells.

However, the essential cellular and molecular targets and a signaling mechanism for resveratrol have not been completely defined. Although type II collagen and sulfated proteoglycan are important for differentiation and COX-2 is significant in the inflammatory response, the underlying regulatory mechanisms of type II collagen and COX-2 in articular chondrocytes are not yet understood. In the present study, the effects of resveratrol on the expression of type II collagen and COX-2 in rabbit articular chondrocytes were investigated and the regulatory mechanisms involved in these effects were investigated.

Resveratrol induces apoptosis or anti-proliferative effects in a variety of cell types, including prostate, breast, lung, leukemia, bladder and ovarian cancer cells (33). It prevents the proliferation of tumor cells by inhibiting DNA synthesis and cell cycle progression and by modulating a series of signaling molecules (17). Resveratrol reduces cell apoptosis and the inflammatory response induced by inflammatory cytokines and inhibits dedifferentiation in arthritic chondrocytes $(30,34)$. In the present study, it was found that resveratrol inhibited the proliferation of rabbit articular chondrocytes (data not shown).

Studies have identified that the diverse effects of resveratrol are regulated differentially according to numerous conditions, such as the concentration of resveratrol and the treatment period (16-18). At a higher dose, resveratrol is pro-apoptotic, inducing apoptosis in cancer cells by exerting a death signal. In addition, at a higher dose, resveratrol depresses cardiac function, elevates the levels of apoptotic protein expression, which results in an unstable redox environment, and increases myocardial infarct size and the number of apoptotic cells (17). The expression levels of proteins associated with cell survival are increased, which results in anti-apoptotic effects, when cells are treated with a low dose of resveratrol (16). Studies have indicated that it may be possible to use resveratrol to prevent and treat OA. For example, Shakibaei et al characterized the effects of IL-1 $\beta$-induced suppression of collagen type II and $\beta 1$-integrin signal receptor synthesis, and observed that the activation of caspase- 3 and PARP cleavage were blocked by resveratrol $(35,36)$. A study has suggested that resveratrol directly blocks caspase- 3 and the subsequent cleavage of PARP and reverses the IL-1 $\beta$-induced upregulation of ROS in chondrocytes (35). Furthermore, resveratrol inhibits the activation of $\mathrm{NF}-\kappa \mathrm{B}$ and thus downregulates $\mathrm{NF}-\kappa \mathrm{B}$-regulated pro-inflammatory gene products such as COX-2, IL-1 $\beta$ and IL-6, which are important in the pathogenesis of OA (37). In the present study, it was demonstrated that a low concentration of resveratrol promotes differentiation, but treatment with a high concentration of resveratrol results in inducement of dedifferentiation (Figs. 1A and 2A), and resveratrol significantly induces the expression of type II collagen in a time-dependent manner (Figs. 1C and 2B). Treatment of rabbit articular chondrocytes with resveratrol was shown to induce the expression of COX-2 and increase $\mathrm{PGE}_{2}$ production in an dose-dependent manner, and the highest expression levels of 
COX-2 and $\mathrm{PGE}_{2}$ production were observed at $3 \mathrm{~h}$ after treatment with resveratrol (Figs. 3 and 4).

MAPK cascades have been shown to be key in the transduction of extracellular signals to cell responses. The MAPK signaling pathways relay, amplify and integrate signals from a wide range of stimuli prior to eliciting an appropriate physiological response that may include cell growth, proliferation, differentiation, development, inflammatory responses, apoptosis and invasion in mammalian cells $(38,39)$. The PI3K/Akt signaling pathway is important for cell growth, differentiation and survival (40). In previous studies, chondrocyte differentiation and the inflammatory response were demonstrated to be associated with the MAPK and PI3K/Akt signaling pathways $(41,29)$. Although the precise mode of resveratrol action has not yet elucidated, a few signaling pathways and molecular targets have been suggested. In several types of tumor cell line, resveratrol has inhibited the activation of JNK and its upstream MAPK/ERK and MEK (14). It has been reported that apoptosis through activation of $\mathrm{p} 53$, which is one of the chemotherapeutic effects of resveratrol, occurs through ERK/p38 (42). In addition, studies have demonstrated that resveratrol inhibits IL-1 $\beta$-induced expression of COX-2 and production of $\mathrm{PGE}_{2}$, causing inhibition of the expression of cartilage-specific collagen type II $(43,44)$. Resveratrol has been shown to induce apoptotic cell death, and suppression of pro-survival PI-3K/Akt signaling may be an important mediator in this process (25). In the present study, resveratrol activated all the ERK, p38, JNK and Akt signaling pathways that belong to the MAPK signaling system (Fig. 5). Therefore, in order to elucidate the association of these signaling systems with cell differentiation and the inflammatory response due to resveratrol, the ERK, p38, JNK and Akt signal transduction pathways were attenuated with their respective inhibitors, PD, SB, SP, TB and LY, following which the expression levels of type II collagen and COX-2 and the synthesized levels of proteoglycan and $\mathrm{PGE}_{2}$ were observed. The results showed that, with the exception of SP treatment, the increased type II collagen and COX-2 expression levels and increased levels of proteoglycan and $\mathrm{PGE}_{2}$ were attenuated following treatment with PD, SB, TB and LY (Figs. 6 and 7).

These results suggest that the differentiation and inflammatory response induced by resveratrol in rabbit articular chondrocytes are regulated through the ERK, p38 and Akt signaling pathways. Since various signaling pathways in addition to the MAPK and Akt signaling pathways, such as the PKC pathway, are associated with the regulation of intrachondrocytic reactions, further detailed studies are required. In addition, as the mechanisms behind the dedifferentiation induced by treatment with a high resveratrol concentration and the suppressed inflammatory response following exposure to resveratrol for long time periods are not known, these also require further investigation. Such study results may be used as fundamental data for the therapy of chondrocytic illnesses such as arthritis.

\section{Acknowledgements}

This study was supported by the Korean Health Technology R\&D Project, Ministry of Health \& Welfare, Republic of Korea (A120960-1201-0000300).

\section{References}

1. Abramson SB, Attur M, Amin AR and Clancy R: Nitric oxide and inflammatory mediators in the perpetuation of osteoarthritis. Curr Rheumatol Rep 3: 535-541, 2001.

2. Araki E, Forster C, Dubinsky JM, Ross ME and Iadecola C: Cyclooxygenase-2 inhibitor NS-398 protects neuronal cultures from lipopolysaccharide-induced neurotoxicity. Stroke 32: 2370-2375, 2001

3. Arichi H, Kimura Y, Okuda H, Baba K, Kozawa M and Arichi S: Effects of stilbene components of the roots of Polygonum cuspidatum Sieb. et Zucc. on lipid metabolism. Chem Pharm Bull (Tokyo) 30: 1766-1770, 1982.

4. Eyre D: Collagen of articular cartilage. Arthritis Res 4: 30-35, 2002.

5. Choy EH and Panayi GS: Cytokine pathways and joint inflammation in rheumatoid arthritis. N Engl J Med 344: 907-916, 2001

6. Héraud F, Héraud A and Harmand MF: Apoptosis in normal and osteoarthritic human articular cartilage. Ann Rheum Dis 59: 959-965, 2000.

7. Kim SJ, Ju JW, Oh CD, et al: ERK-1/2 and p38 kinase oppositely regulate nitric oxide-induced apoptosis of chondrocytes in association with p53, caspase-3, and differentiation status. J Biol Chem 277: 1332-1339, 2002.

8. Sandell LJ and Adler P: Developmental patterns of cartilage. Front Biosci 4: D731-D742, 1999.

9. Sandell LJ and Aigner T: Articular cartilage and changes in arthritis. An introduction: cell biology of osteoarthritis. Arthritis Res 3: 107-113, 2001.

10. Hawkey CJ: COX-2 inhibitors. Lancet 353: 307-314, 1999.

11. Pecchi E, Priam S, Mladenovic Z, et al: A potential role of chondroitin sulfate on bone in osteoarthritis: inhibition of prostaglandin $\mathrm{E}_{2}$ and matrix metalloproteinases synthesis in interleukin-1 $\beta$-stimulated osteoblasts. Osteoarthritis Cartilage 20: 127-135, 2012.

12. Kim SJ, Im DS, Kim SH, et al: Beta-catenin regulates expression of cyclooxygenase-2 in articular chondrocytes. Biochem Biophys Res Commun 296: 221-226, 2002.

13. Khanna D, Sethi G, Ahn K S, et al: Natural products as a gold mine for arthritis treatment. Curr Opin Pharmacol 7: 344-351, 2007.

14. Shakibaei M, Harikumar KB and Aggarwal BB: Resveratrol addiction: to die or not to die. Mol Nutr Food Res 53: 115-128, 2009.

15. Liao PC, Ng LT, Lin LT, Richardson CD, Wang GH and Lin CC: Resveratrol arrests cell cycle and induces apoptosis in human hepatocellular carcinoma Huh-7 cells. J Med Food 13: 1415-1423, 2010.

16. Mukherjee S, Dudley JI and Das DK: Dose-dependency of resveratrol in providing health benefits. Dose Response 8: 478-500, 2010.

17. Hwang JT, Kwak DW, Lin SK, Kim HM, Kim YM and Park OJ: Resveratrol induces apoptosis in chemoresistant cancer cells via modulation of AMPK signaling pathway. Ann NY Acad Sci 1095: 441-448, 2007.

18. Lin HY, Sun M, Tang HY, et al: Resveratrol causes COX-2- and p53-dependent apoptosis in head and neck squamous cell cancer cells. J Cell Biochem 104: 2131-2142, 2008.

19. Lin HY, Tang HY, Davis FB and Davis PJ: Resveratrol and apoptosis. Ann NY Acad Sci 1215: 79-88, 2011.

20. Yu W, Fu YC and Wang W: Cellular and molecular effects of resveratrol in health and disease. J Cell Biochem 113: 752-759, 2012.

21. Elmali N, Esenkaya I, Harma A, Ertem K, Turkoz Y and Mizrak B: Effect of resveratrol in experimental osteoarthritis in rabbits. Inflamm Res 54: 158-162, 2005.

22. Meeran SM and Katiyar SK: Cell cycle control as a basis for cancer chemoprevention through dietary agents. Front Biosci 13: 2191-2202, 2008

23. Bäckesjö CM, Li Y, Lindgren U and Haldosén LA: Activation of Sirtl decreases adipocyte formation during osteoblast differentiation of mesenchymal stem cells. J Bone Miner Res 21: 993-1002, 2006.

24. Banerjee Mustafi S, Chakraborty PK and Raha S: Modulation of Akt and ERK1/2 pathways by resveratrol in chronic myelogenous leukemia (CML) cells results in the downregulation of Hsp70. PLoS One 5: e8719, 2010.

25. Jiang $\mathrm{H}$, Shang $\mathrm{X}, \mathrm{Wu} \mathrm{H}$, et al: Combination treatment with resveratrol and sulforaphane induces apoptosis in human U251 glioma cells. Neurochem Res 35: 152-161, 2010. 
26. Weng $\mathrm{CJ}, \mathrm{Wu} \mathrm{CF}$, Huang $\mathrm{HW}, \mathrm{Wu} \mathrm{CH}$, Ho $\mathrm{CT}$ and Yen $\mathrm{GC}$ : Evaluation of anti-invasion effect of resveratrol and related methoxy analogues on human hepatocarcinoma cells. J Agric Food Chem 58: 2886-2894, 2010.

27. Csaki C, Mobasheri A and Shakibaei M: Synergistic chondroprotective effects of curcumin and resveratrol in human articular chondrocytes: inhibition of IL-1beta-induced NF-kappaB-mediated inflammation and apoptosis. Arthritis Res Ther 11: R165, 2009.

28. Yoon YM, Kim SJ, Oh CD, et al: Maintenance of differentiated phenotype of articular chondrocytes by protein kinase $\mathrm{C}$ and extracellular signal-regulated protein kinase. J Biol Chem 277: 8412-8420, 2002.

29. Blanco FJ, Guitian R, Moreno J, de Toro FJ and Galdo F: Effect of antiinflammatory drugs on COX-1 and COX-2 activity in human articular chondrocytes. J Rheumatol 26: 1366-1373, 1999.

30. Lei M, Liu SQ and Liu YL: Resveratrol protects bone marrow mesenchymal stem cell derived chondrocytes cultured on chitosan-gelatin scaffolds from the inhibitory effect of interleukin-1beta. Acta Pharmacol Sin 29: 1350-1356, 2008.

31. Tang FY, Su YC, Chen NC, Hsieh HS and Chen KS: Resveratrol inhibits migration and invasion of human breast-cancer cells. Mol Nutr Food Res 52: 683-691, 2008.

32. Gweon EJ and Kim SJ: Resveratrol induces MMP-9 and cell migration via the p38 kinase and PI-3K pathways in HT1080 human fibrosarcoma cells. Oncolgy Rep 29: 826-834, 2013.

33. Hashimoto S, Ochs RL, Komiya S and Lotz M: Linkage of chondrocyte apoptosis and cartilage degradation in human osteoarthritis. Arthritis Rheum 41: 1632-1638, 1998.

34. Shakibaei M, Mobasheri A and Buhrmann C: Curcumin synergizes with resveratrol to stimulate the MAPK signaling pathway in human articular chondrocytes in vitro. Genes Nutr 6: 171-179, 2011.
35. Csaki C, Keshishzadeh N, Fischer K and Shakibaei M: Regulation of inflammation signalling by resveratrol in human chondrocytes in vitro. Biochem Pharmacol 75: 677-687, 2008.

36. Shakibaei M, John T, Seifarth C and Mobasheri A: Resveratrol inhibits IL-1 beta-induced stimulation of caspase- 3 and cleavage of PARP in human articular chondrocytes in vitro. Ann NY Acad Sci 1095: 554-563, 2007

37. Wang J, Gao JS, Chen JW, Li F and Tian J: Effect of resveratrol on cartilage protection and apoptosis inhibition in experimental osteoarthritis of rabbit. Rheumatol Int 32: 1541-1548, 2012.

38. Widmann C, Gibson S, Jarpe MB and Johnson GL: Mitogen-activated protein kinase: conservation of a three-kinase module from yeast to human. Physiol Rev 79: 143-180, 1999.

39. Zhang W and Liu HT: MAPK signal pathways in the regulation of cell proliferation in mammalian cells. Cell Res 12: 9-18, 2002

40. Foster FM, Traer CJ, Abraham SM and Fry MJ: The phosphoinositide (PI) 3-kinase family. J Cell Sci 116: 3037-3040, 2003.

41. DeLise AM, Fischer L and Tuan RS: Cellular interactions and signaling in cartilage development. Osteoarthritis Cartilage 8: 309-334, 2000.

42. She QB, Bode AM, Ma WY, Chen NY and Dong Z: Resveratrol-induced activation of p53 and apoptosis is mediated by extracellular-signal-regulated protein kinases and p38 kinase. Cancer Res 61: 1604-1610, 2001

43. Wight RD, Tull CA, Deel MW, et al: Resveratrol effects on astrocyte function: relevance to neurodegenerative diseases. Biochem Biophys Res Commun 426: 112-115, 2012.

44. Shakibaei M, Csaki C, Nebrich S and Mobasheri A: Resveratrol suppresses interleukin-1beta-induced inflammatory signaling and apoptosis in human articular chondrocytes: potential for use as a novel nutraceutical for the treatment of osteoarthritis. Biochem Pharmacol 76: 1426-1439, 2008 